
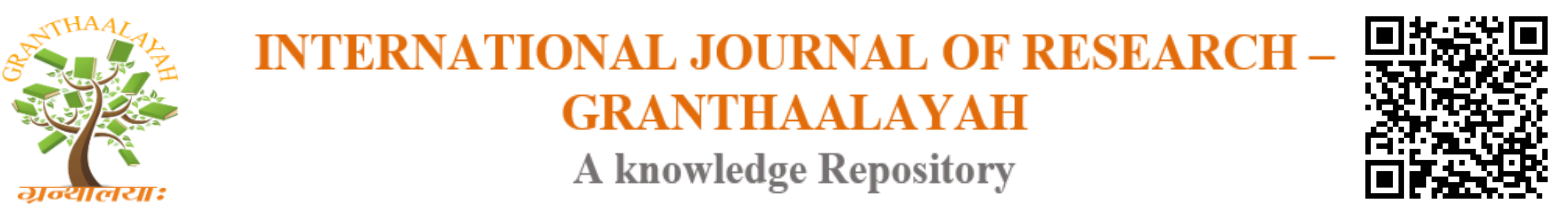

Science

\title{
PRESUMPTIVE DIAGNOSIS OF MALARIA IN FEBRILE CHILDREN; IS IT JUSTIFIED?
}

\author{
Animasahun Adeola B ${ }^{* 1}$, Adekunle Motunrayo O², Afadapa Maria A ${ }^{3}$, \\ Salisu Mohammed A \\ *1 Associate Professor, Department of Paediatrics and Child Health, Lagos State University \\ College of Medicine, Ikeja Lagos Nigeria \\ ${ }^{2}$ Consultant Paediatrician, Department of Paediatrics and Child Health, Lagos State University \\ College of Medicine, Ikeja Lagos Nigeria \\ ${ }^{3}$ Senior Medical Officer, Department of Paediatrics and Child Health, Lagos State University \\ College of Medicine, Ikeja Lagos Nigeria \\ ${ }^{4}$ Department of Paediatrics and Child Health, Lagos State University College of Medicine \\ Ikeja Lagos Nigeria
}

\begin{abstract}
Background: Malaria is deadly and a major disease burden in Africa especially among the under-5s. Due to the high death rate from malaria in endemic region, World Health Organization previous recommendation was presumptive treatment of malaria.

Aim: This study aimed to evaluate if presumptive diagnosis of malaria in our region is still justified, and increase the awareness for proper evaluation for other causes of fever in febrile children.

Methods: Apart of a single blinded prospective interventional study carried out between March and May 2016, at the children outpatient unit of Lagos State University Teaching Hospital, Ikeja, Nigeria. Consecutive children aged 6 months to 12 years with temperature of 37.50C and above at presentation without prior antimalaria treatment were recruited. Clinical and laboratory evaluation for malaria were done in these subjects. Data were analyzed using the Statistical Package for Social Sciences (SPSS) version 20.0. Test of statistical significant was set at $<0.05$.

Results: Eighty-eight (88) children were recruited. The mean age of studied subjects was $43 \pm 46.5$ months with a median of 25 months. Male to female ratio was 1.1:1. The axillary temperature range of studied subjects ranged between $37.50 \mathrm{C}$ to $390 \mathrm{C}$. The mean temperature of subjects was $37.8 \pm 0.370 \mathrm{C}$. Out of 88 children recruited for the study, 51 (58\%) had microscopic malaria test being positive. Up to 37 (42\%) febrile children tested negative for malaria.

Conclusion: Malaria infection in children is still high in our environment, but presumption treatment of malaria is no longer justified. There is a need to scale up provision of diagnostic tools for malaria so that overtreatment can be curtailed.
\end{abstract}

Keywords: Malaria; Fever; Presumptive Treatment; Nigeria. 
Cite This Article: Animasahun Adeola B, Adekunle Motunrayo O, Afadapa Maria A, and Salisu Mohammed A. (2017). "PRESUMPTIVE DIAGNOSIS OF MALARIA IN FEBRILE CHILDREN; IS IT JUSTIFIED?." International Journal of Research - Granthaalayah, 5(12), 291-297. https://doi.org/10.29121/granthaalayah.v5.112.2017.505.

\section{Introduction}

Malaria is a deadly disease; it is a major disease burden in Africa especially among the under-5s [1] the burden of malaria infection and its causal effect is concentrated in the sub-Saharan countries where over $88 \%$ of the cases occur with $90 \%$ death occurrence in this region. [1]

In Nigeria, the burden of malaria is high as $97 \%$ among the population at risk. [2] It account for over $60 \%$ of public hospital visit. [2] Malaria is the commonest cause of morbidity and mortality in Nigerian children accounting for $20 \%$ of mortality in under-5s.[2] Despite this data, there was appreciable decline in the occurrence of malaria by $18 \%$ in the year 2015 in Nigeria.[3]

Due to the high death rate from malaria in endemic region, World Health Organization previous recommendation was presumptive treatment of malaria. [4] However, the availability of rapid diagnostic test has led the recommendation of parasitological diagnosis before treatment. [5]Reasons for the new guideline are the recorded successes of decline in malaria transmission in sub-Saharan Africa and the availability of rapid diagnostic test. [5] However, there is a conflicting opinion by Graz et al. [6] where the cost effectiveness of parasitological diagnosis before treatment of malaria infection especially in regions yet to attain a decline in infection rate was questioned.

The benefits of testing before treatment of malaria have been highlighted by World Health Organization; [5] this will prevent unnecessary use of antimalaria and will reduce the frequency of adverse effect especially in those that do not need the drug. Also, test before treatment will improve malaria case detection and reporting.

In five primary health centers in Jos, Nigeria, Ikeh and co-researchers [7] found a prevalence of malaria of $56.9 \%$ among 260 under-five children with fever. The prevalence of malaria in subjects that have not had antimalaria prior to presentation was $56.8 \%$ and a slightly higher prevalence of $57 \%$ was reported in subjects that had antimalaria commenced before presentation. Prevalence of malaria in febrile children presumptively diagnosed with malaria was $54 \%$ in a study done in Zamfara, Nigeria by Abdulkadir et al. [8] However, in this study, 57\% of the subjects already had one form of antimalaria treatment.

There is need for further review of the routine presumptive malaria diagnosis which currently is still being practiced in most centers in Nigeria. This will provide knowledge on the need for further review of malaria protocols and guidelines and also provide additional data on the effectiveness of measures instituted in the eradication of malaria. This study aimed to evaluate the rightfulness of presumptive diagnosis of malaria in our region and increase the awareness for proper evaluation for other causes of fever in febrile children. 


\section{Subjects and Methods}

This was part of a single blinded prospective interventional study carried out between March and May 2016 at the outpatient unit of Lagos State University Teaching Hospital, Ikeja, Nigeria (LASUTH). It is a center that receives patients from both public and private clinics within Lagos State and it's environ. The Paediatric outpatient department of LASUTH attends to averagely 80 children per day.

Inclusion criteria for the study was children aged 6 months to twelve years with fever of temperature of $37.5^{\circ} \mathrm{C}$ and above at presentation who have not had any antimalaria treatment in the current illness. Informed and written consent was obtained from the caregiver and assent was obtained from children seven years and above before recruitment into the study. Clinical and laboratory evaluation for malaria was done in all the children with febrile illness: Throat, ear and other systemic examinations were done for the identification of the cause of the fever. As required, further investigations done were chest radiograph and swab analysis for microscopy culture and sensitivity in cases with suppurative otitis media. Microscopy test for malaria was done in children that met the inclusion criteria. Microscopy test for malaria was done in children that met the inclusion criteria. Examination for the presence of malaria parasite density and specie was determined using the thick film and the thin blood film respectively. The thin film was fixed in methanol. Staining of the film using a Giemsa stain and visual examination using a microscope was done. The red blood cells were examined for intracellular parasites. Semi quantitative count of parasitized red blood cell using the plus system was done. The presence of 1 to 10 and 11 to 100 asexual parasites per 100 thick field was classified as one plus (+) and 2 pluses (++) respectively. 1 to 10 and 11 to 100 asexual parasites per one thick field was classified as $3(+++)$ and $4(++++)$ pluses respectively. Thorough examination of at least 100 microscopic field of thick film was examined before a result was concluded as negative result. The thin films were examined for different morphologic features of the different species of malaria parasite. [9] Complete blood count was also done. Antimalaria medication was given to children that tested positive for malaria and a repeat microscopy malaria test was done one week after completion of the antimalaria to ensure parasitic clearance.

Data were analyzed using the Statistical Package for Social Sciences (SPSS) version 20.0.Patients' demographics were represented as frequency and percentages. Continuous variables were presented as mean \pm standard deviation for parametric data. Test of statistical significant was set at $<0.05$.

\section{Results}

A total number of 88 children had fever at presentation during the study period. The mean age of studied subjects was $43 \pm 46.5$ months with a median value of 25 months. The median age was 25 months with the highest number of subjects being 12 months of age. Male subjects seen were $53 \%$ and female accounted for $47 \%$ of the studied subjects. The axillary temperature range of studied subjects was $37.5^{\circ} \mathrm{C}$ to $39^{\circ} \mathrm{C}$. The mean temperature of subjects was $37.8 \pm 0.37^{\circ} \mathrm{C}$ and the median temperature was $37.7^{\circ} \mathrm{C}$ 
Out of 88 children recruited for the study, 51 had microscopic malaria test being positive (58\%) and $42 \%$ febrile children tested negative for malaria. Malaria infection was highest within the age range of 6 months and five years which accounted for $71 \%$ of positively malaria tested subjects. Only four subjects $(7.8 \%)$ with positive result were between six and twelve months.

Table 1: Demographic Characteristics of Subjects

\begin{tabular}{|l|l|l|}
\hline Sex Distribution of Subjects & Male & $47(53 \%)$ \\
\cline { 2 - 3 } & Female & $41(47 \%)$ \\
\hline Age Distribution of Subjects with Malaria & Less than 1 year & $4(7.8 \%)$ \\
\cline { 2 - 3 } & Years & $34(66.7 \%)$ \\
\cline { 2 - 3 } & >5 years & $13(25.5 \%)$ \\
\hline
\end{tabular}

All children that had parasitological diagnosis for malaria had Plasmodium falciparum as the causative parasite and they all had one plus of malaria parasite (+) which is 1 to 10 parasitized red cells per 100 thick fields. They all had artemisinin combination therapy for three days and a repeat microscopic test done in 40 out of 51 children. Eleven children treated for malaria infection were lost to follow-up. Repeat parasitological test in 40 that presented for follow up showed total clearance of parasite in the blood film.

Result of full blood count in four children treated for malaria showed co-bacterial infection evidenced by leukocytosis and neutrophilia. The mean packed cell volume of subjects with malaria was $31.7 \pm 4.9 \%$.

In children with negative result for malaria parasite, 54\% had upper respiratory tract infection and 3 case of uncomplicated bronchopneumonia were seen. A five years old boy had diagnosis of mumps following subsequent development of jaw swelling. Other causes of fever diagnosed were acute suppurative otitis media, diarrhea disease, tonsillitis, urinary tract infection and bronchiolitis.

Table 2: Diagnosis in Subjects

\begin{tabular}{|l|l|}
\hline Disease Conditions & No of Cases (\%) \\
\hline Malaria & $51(58 \%)$ \\
\hline URTI & $20(22.7 \%)$ \\
\hline $\begin{array}{l}\text { Uncomplicated } \\
\text { Bronchopneumonia }\end{array}$ & $3(3.4 \%)$ \\
\hline Suppurative Otitis Media & $2(2.3 \%)$ \\
\hline Bronchiolitis & $2(2.3 \%)$ \\
\hline Furunculosis & $2(2.3 \%)$ \\
\hline Diarrheoa & $1(1.1 \%)$ \\
\hline Mumps & $1(1.1 \%)$ \\
\hline Tonsillitis & $1(1.1 \%)$ \\
\hline Urinary Tract Infection & $1(1.1 \%)$ \\
\hline Sepsis & $1(1.1 \%)$ \\
\hline Unspecified & $3(3.4 \%$ \\
\hline
\end{tabular}




\section{Discussion}

The prevalence of malaria infection in febrile children aged 6 months to 12 years in the current study is $58 \%$. This is similar to the findings by Ikeh et al. [7] and Abdulkadir et al. [8] with result of $54 \%$ and $56.3 \%$ respectively. A similar finding was also seen in the study done between October 2008 and June 2009 in Sokoto, Nigeria by Singh et al. [10] where the prevalence of malaria in 800 febrile children was 56\%.However, the prevalence in the current study is higher than a prevalence of $20.7 \%$ reported by Oladosu and Oyibo[11] in a primary health center in Lagos. Inclusion of children without fever at presentation could account for a lower prevalence in the their study The prevalence in this present study and other Nigerian studies reviewed above are high compared to a prevalence of $12 \%$ seen by Mazigo et al. [12] in Tanzania. This disparity is alarming despite both regions with holo-endemicity for malaria. The immediate reason for this disparity is not clear.

Presumptive diagnosis of malaria in febrile children is commonly done in malaria endemic region. In this present study, $42 \%$ of the subjects would have been wrongly diagnosed and treated for malaria. This is lower than the finding by Okoro et al. [13] in Imo State, Nigeria where $92.6 \%$ of subjects that had fever had negative blood film for malaria parasite. The inclusion of children without fever at presentation but within its 24 hour could have falsely increased the number of children with fever. Children recruited in this study were those with fever at presentation. This is because tactile assessment of fever is often practiced in our environment which has been studied to overestimate fever by over $24 \%$. [14]

Presumptive diagnosis of malaria in febrile children is high in our environment. This has led to unnecessary treatment with antimalaria with manifestations of adverse effect. Omission of life threatening causes or complications of other causes of fever in children can occur if children are presumptively treated as having malaria. Clinical diagnosis of malaria has a low specificity and overtreatment of cases often occur. [5] Though the need for proper evaluation of every child for other causes of fever is the standard practice, in region were children are presumptively treated for malaria, this is often neglected. [13]

The existence of rapid diagnostic testing for malaria has made quick and universal laboratory screening and diagnosis of malaria a possibility. Rapid diagnostic test has a high sensitivity for the detection of malaria parasites. The confirmation of infection in those with possible falsely negative result can then be confirmed by microscopic testing. [8]

Malaria was commonest in subjects five years and below. This is not surprising because there is deficient immunity for malaria parasite in this age group. This finding is similar to that reported by other researchers in Nigeria. However, this finding has not been consistent; Umaru and coresearchers [15] had the highest prevalence of malaria in children five to fifteen years. No immediate explanation can be given for this conflicting result.

Subjects with positive malaria test in infancy were only7.8\% of the subjects. The explanation for this is the protection from malaria due to the presence of passive immunity acquired from the mother. This finding was also seen in the studies by Mbanugo et al. [16] and Nwaorgu et al. [17] 
No significant difference was seen in the prevalence of malaria between male and female subjects with a disparity of slightly higher percentage in male subjects. This is similar to the result reported by Nmadu et al. [18] were there was a slight male preponderance.

Plasmodium falciparum was the causative parasite of malaria in all subjects in this current study. This finding is an additional report to previously documented findings of Plasmodium falciparum as the commonest Plasmodium specie that causes malaria with a high predominance in Africa. $[7,16,19]$ The predominance of P. falciparum parasite, the deadliest specie in Nigeria emphasizes the need for drastic measures for eradication of malaria.

Artemisinin combination therapy is the WHO recommended choice of drug for uncomplicated antimalaria. [5] In this present study there was $100 \%$ clearance of parasite in the blood following the use of the combination therapy.

The second commonest cause of fever in this current study is upper respiratory tract infection. Subjects with this diagnosis had mainly coryza and mild fever. The explanation for this is possible because the study was done in rainy season when respiratory tract infection occurrence is high. TemiyeEdamisan [20] identified malaria and respiratory infection as the commonest illnesses in the rainy period. Increase in the breeding sites of mosquito occurs at this period, and the tendency for closure of windows at this period with inadequate ventilation increases the spread of germs that causes respiratory infection.

\section{Conclusion}

Malaria infection in children is still high in our environment. Plasmodium Falciparum is still the predominant cause. Presumptive treatment of malaria can no longer be justified andrequires urgent attention. There is need for a scale up in provision and accessibility of diagnostic tools for malaria so that overtreatment can be curtailed. The need for more preventive measures in control of malaria infection cannot be overemphasized.

\section{Ethical disclosures}

Ethical clearance for the study was obtained. Informed consent was obtained from the parent.

\section{Acknowledgement}

We gratefully acknowledge the subjects who participated in this study, their parents, caregivers and other healthcare workers involved in their care.

\section{Disclosures}

No Conflict of interest among the authors

\section{References}

[1] World Health Organization. World Malaria Report.2015. http//www.who.int (Last Accessed 03 September 2016) 
[2] President's Malaria Initiative Nigeria. Malaria Operational Plan FY 2015.

[3] President's Malaria Initiative Strategy. https://www.pmi.gov/ (Last Accessed 03 September, 2016)

[4] WHO: Guidelines for the treatment of malaria 2006, Geneva: WHO.

[5] WHO: Guidelines for the treatment of malaria (2e).2010, Geneva: World Health Organization, 2.

[6] Graz B, Willcox M, Szeless T, Rougemont A. "Test and Treat" or presumptive treatment for malaria in high transmission situation? A reflection on the latest WHO guidelines. Malaria Journal 2011, 10:136.

[7] Ikeh EI, Teclaire NN. Prevalence of malaria parasitemia and associated factors in febrile under5 chidren seen in Primary Health Centres in Jos, North Central Nigeria. Niger Postgrad Med J, 2008 Jun;15(2):65-9

[8] Abdulkadir I, Rufai HA, Ochapa SO, Malam MS, Garba BI, Oloko AY, George II. Malaria rapid diagnostic test in children: The Zamfara, Nigeria experience. Niger Med J 2015[serial online] 2015[cited 2016 Jun 26]; 56:278-82.

[9] WHO. Basic Malaria Microscopy Part1. Learner's guide; Part II Tutor's guide. Geneva, World Health Organization, 1991.

[10] Singh S, Madaki AJ, Jiya NM, Singh R, Tracher TD. Predictors of malaria in febrile children in Sokoto, Nigeria. Niger Med J 2014 Nov-Dec;55(6):480-485.

[11] Oladosu OO, Oyibo WA. Overdiagnosis and overtreatment of malaria in children that presented with fever in Lagos, Nigeria. ISRN Infectious Diseases.2013;2013:1-6

[12] Mazigo HD, Meza W, Ambrose EE, Kidenya BR, Kweka EJ. Confirmed malaria cases among children under five with fever and history of fever in rural western Tanzania. BMC Research. 2011; 359:1-6.

[13] Okoro CI, Chukwuocha UM, Nwakwuo GC, Ukaga CN. Presumptive Diagnosis and Treatment of Malaria in Febrile Children in Parts of South Eastern Nigeria. J Infect Dis Ther. 2015; 3:5

[14] Odinaka KK, Edelu BO, Nwolisa EC, Amamilo IB, Okolo SN. Accuracy of subjective assessment of fever by Nigerian mothers in under-5 children. Niger Med J. 2014; 55(4):338-341.

[15] Umaru ML, Uyaiabasi GN. Prevalence of Malaria in Patients Attending the General Hospital Makarfi, Makarfi Kaduna-State, North Western Nigeria. American Journal of Infectious Diseases and Microbiology .2015;13(1):1-5.

[16] Mbanugoji, Ejim DO. Plasmodium Infections in Children Aged 0-5Yrs in Akwa Metropolis, Anambra State, Nigeria. Nigerian J of Parasitology. 2000; 21:55-59

[17] Nwaorgu OC, Orajaka BN. Prevalence of Malaria among Children 1-10 Years Old in Communities in Akwa North Local Government Area, Anambra State South East Nigeria. International Multidisciplinary Journal Ethiopia;5(5) serial No 22, Oct, 2011:264-281.

[18] Nmadu PM, Peter E, Alexander AZ, Koggie AZ, Maikenti JI. The Prevalence of Malaria in Children between the Ages 2-15 visiting Gwarinpa General Hospital Life-Camp, Abuja, Nigeria. Journal of Health Science. 2015;5(3):47-51

[19] The public health burden of plasmodium falciparum malaria in Africa: Deriving the numbers by Robert W Snow, Merlies H Craig, Charles R.J.C. Newton, and Richard W. Steketer. Working paper No. 11, Disease control priorities project. Bethesda, Maryland: Fogarty International Center, National Institutes of Health, August 2003.

[20] Temiye Edamisan. Paediatrician says respiratory infection, malaria major illnesses in children during rainy season. In News Agency of Nigeria Jun 25, 2015.

*Corresponding author.

E-mail address: deoladebo@yahoo.com/motunbamm@yahoo.com/departmentofpaediatrics@ gmail.com/ wolebamgbala@gmail.com

Phone number: +2348037250264 